\title{
Estrus Induction by Hormonal and Nutritional Approaches in Canines
}

\author{
M. Honparkhe ${ }^{1}$, Jugraj Singh Mahal $^{2} *$ and Ajeet Kumar ${ }^{1}$ \\ ${ }^{1}$ Department of Veterinary Gynaecology and Obstetrics, Guru Angad Dev Veterinary and \\ Animal Sciences University, Ludhiana, Punjab, India \\ ${ }^{2}$ Department of veterinary Gynaecology and Obstetrics, Khalsa College of Veterinary and \\ Animal sciences, Amritsar, Punjab, India \\ *Corresponding author
}

\begin{abstract}
A B S T R A C T
The present study was conducted to compare the efficacy of hormonal and nutritional approaches to induce fertile estrus in canine. Twenty female dogs of different breeds presented to university clinics, GADVASU, Ludhiana were subjected to hormonal (Group 1, $\mathrm{n}=10$ ) and nutritional (Group 2; $\mathrm{n}=10$ ) treatments. The breeds of these dogs were Labrador, Dalmatian and German Shephard weighing between 25-30 kg with the history of not showing estrus despite attaining age of puberty ( $\geq 1.5$ years of age). Prior to start of any treatment, all the animals were subjected to deworming and transabdominal ultrasonography of reproductive organ to assess uterine development. Hormonal treatment included eCG @ 5 IU/kg (Inj Folligon; MSD Intervet) daily for 10 days followed by hCG $500 \mathrm{IU}$ (Inj Chorulon, MSD Intervet) intramuscularly on day 11. Nutritional treatment involved oral administration of tab Nutrich (Virbac, India) @ one tab daily for 15 days and tab Evion LC (Merk India) @ 1 tab daily for 15 days one month before start of breeding season. Following hormonal treatment majority of Group 1 female dogs (7/10) exhibited proestrus bleeding at 5-6 days of eCG treatment which was confirmed by exfoliative vaginal cytology indicating abundant parabasal cells, few large and small intermediate cells and RBCs. On day 11, these animals were again subjected to vaginal cytology to confirm presence of abundant superficial cells (>80\%). These dogs were advised for mating twice at 24 and $48 \mathrm{hrs}$ after hCG administration. Two female dogs from group 1 encountered with the problem of ovarian cysts whereas one had developed cystic endometrial hyperplasia. Group 2 animals exhibited proestrus bleeding (5/10) during subsequent breeding season and were subjected to vaginal cytology twice (i.e. first at 4 days after start of bleeding and second next day after the end of bleeding). These animals were advised for mating when $>80 \%$ superficial cells were observed in vaginal cytology. Following treatment, five and four animals conceived respectively in groups 1 and 2 . The results indicated that hormonal and nutritional approaches are equally good in inducing fertile estrus in canine.
\end{abstract}

\section{Introduction}

The dogs are non-seasonal monoestrous animals. Most of the breeds having one breeding cycle in a season. Normally bitches attain puberty at an age of 6-14 months and there starts first estrous cycle. Each estrous cycle is divided into four phases such as proestrus, estrus, diestrus and anestrus. Different signs are observed during each phase related to clinical, hormonal, behaviour, physiological ad cytological changes in canines.

The anestrus is considered as longest phase of estrous cycle in bitches which usually varies 
from 100-150 days and characterized by smooth, inactive ovaries and no discharges from vulva. The bitches may be either in primary or secondary anestrus. Primary anestrus is usually considered when the first estrus has not occurred upto 2 years of age. There are various factors of primary anestrus which includes abnormality of sexual differentiation, ovarian aplasia, ovariectomy, progesterone secreting ovarian cysts hypothyroidism etc (Sontas et al., 2014). The secondary anestrus is termed when first estrus occurred normally but consecutive second estrus takes around 18 months in reoccurrence (Rosychuk et al., 1983 and Peterson et al., 1997).

The main causes of secondary anoestrus are hypothyroidism, stress and endocrine abnormalities. The presence of cornified cells of vaginal epithelium for 21 to 28 days due to production of endogenous estrogen or use of exogenous estrogen is defined as persistent estrus (Perkins and Thomas, 1993). The follicular cysts, tumour of granulose cells and exogenous use of estrogen are the main causes of persistent estrus. The period of sexual receptivity is called estrus (Ribeiro et $a l ., 2007)$. The level of progesterone in blood greater than $2.0 \mathrm{ng} / \mathrm{mL}$ on monthly basis is an indicator of functional ovary (Clark and Stainer, 1983).

In vaginal smears, the relative ratio of different cell types can be used to identify the hormonal status which helps in detecting the phase of the estrous cycle (Ribeiro et al., 2007). Most approaches for estrus induction in the bitch suffer from poor results and many have not been tested sufficiently to permit recommendation for clinical use (Concannon, 2002). The estrus in female dogs is mainly induced by the drugs that are prolactin inhibitors, both dopamine agonists and serotonin antagonists, prostaglandins and their analogs, GnRH, agonists and superagonists of
GnRH, gonadotropins, and estrogens. The efficacy in inducing fertile estrous varies according to the phase of estrous cycle in which the treatment is conducted and the protocol used (Kutzler, 2005).

\section{Materials and Methods}

The present study was conducted to compare the efficacy of hormonal and nutritional approaches to induce fertile estrus in canine. Twenty female dogs of different breeds presented to university clinics, GADVASU, Ludhiana were subjected to hormonal (Group 1, $\mathrm{n}=10$ ) and nutritional (Group 2; $\mathrm{n}=10$ ) treatments. The breeds of these dogs were Labrador, Dalmatian and German Shephard weighing between $25-30 \mathrm{~kg}$ with the history of not showing estrus despite attaining age of puberty ( $\geq 1.5$ years of age).

So, all the bitches were considered under the Primary anestrus. Many protocols were used to induce estrus in female dogs. However, these have not been sufficiently tested to become common practice (Concannon and Verstegen 1997). Prior to start of the treatment, all the animals were subjected to deworming and transabdominal ultrasonography of reproductive organ to assess uterine development.

Hormonal treatment included eCG @ $5 \mathrm{IU} / \mathrm{kg}$ (Inj Folligon; MSD Intervet) daily for 10 days followed by hCG 500 IU (Inj Chorulon, MSD Intervet) intramuscularly on day 11 . Nutritional treatment involved oral administration of tab Nutrich (Virbac, India) @ one tab daily for 15 days and tab Evion LC (Merk India)@1 tab daily for 15 days one month before start of breeding season. Vaginal cytology is most appropriate method in bitches compared to other animals to differentiate stages of estrous cycles. The vaginal cytology of female dogs from both groups were evaluated to determine anestrus. 
The objective was to obtain a sample of epithelial cells from the vagina.

The sterile cotton swabs were inserted through the cranial end of vagina, which allowed the cotton tip to pick up an adequate number of cells. The smear was immediately prepared after withdrawal of the swab by rolling the cotton tip on the glass microscope slide. As soon as the smear was prepared, the microscopic slides were dipped 5 to 10 times in a container of methanol and stained with Giemsa stain.

The slides were viewed using an electrical microscope, with 100-x (oil immersion) magnification, to assess the cell types of the vaginal epithelium. When vaginal cytology showed characteristics of low cellularity, with a predominance of parabasal cells, the female dogs were subjected to hormonal treatment.

Majority of Group 1 female dogs (7/10) exhibited proestrus bleeding at 5-6 days of eCG treatment which was confirmed by exfoliative vaginal cytology indicating abundant parabasal cells, few large and small intermediate cells and RBCs. The first day of pro-oestrus was considered when there was bloody discharge, vulval swelling and $>20 \%$ intermediate or cornified epithelial cells in the vaginal smear. On day 11, these animals were again subjected to vaginal cytology to confirm presence of estrus phase. Estrus was confirmed by abundant superficial cells $(>80 \%)$ which were displaying pyknotic nuclei, combined with the behavioral characteristic of this phase. These dogs were advised for mating twice at 24 and $48 \mathrm{hrs}$ after hCG administration.

Group 2 animals exhibited proestrus bleeding $(5 / 10)$ during subsequent breeding season and were subjected to vaginal cytology twice (i.e. first at 4 days after start of bleeding and second next day after the end of bleeding). These animals were advised for mating when
$>80 \%$ superficial cells were observed in vaginal cytology.

The female dogs were then mated with males of their respective breeds, by natural mating.

\section{Results and Discussion}

Following hormonal treatment majority of Group 1 female dogs (7/10) exhibited proestrus bleeding at 5-6 days of eCG treatment which was confirmed by exfoliative vaginal cytology indicating abundant parabasal cells, few large and small intermediate cells and RBCs. On day 11, these animals were again subjected to vaginal cytology to confirm presence of abundant superficial cells $(>80 \%)$. These dogs were advised for mating twice at 24 and $48 \mathrm{hrs}$ after hCG administration.

Two female dogs from group 1 encountered with the problem of ovarian cysts whereas one had developed cystic endometrial hyperplasia. The IM administration of eCG at the dose of $20 \mathrm{IU} / \mathrm{kg} /$ day, for 10 days during anestrus and followed by hCG (500-1000 IU), results in estrus in bitches (Wright, 1982). However, such a therapy is frequently characterized by low fertility, hyperestrogenism, cystic ovaries, anovulation, inadequate luteal phase and/or pregnancy resorption (concannon, 2002). The same eCG doses given for 5 days and immediately followed by hCG resulted in high fertility in some studies (Arnold et al., 1989) but not in other (Concannon, 2002).

Factors complicating the use of this protocol include potential differences among preparations of eCG and biopotency estimates, and availability in different countries (Concannon, 2002). Other doses, frequencies or durations have been recently reviewed (Kutzler, 2005) and it was mentioned that the ovulation and pregnancy rates were low or not evaluated in most 
studies. Alternate day eCG administration did not induce proestrus in bitches (van Haaften et al., 1989; Verstegen et al., 1997) conducted a study on the termination of obligate anoestrus and induction of fertile ovarian cycles in dogs by administration of purified pig $\mathrm{LH}$ and opined that increased $\mathrm{LH}$ alone can initiate a competent follicular phase in bitches.

Furthermore, functional pro-oestrus-like follicle development apparently does not require increases in FSH beyond the increased values already present throughout most of anoestrus. However, the purified LH preparation used in the study was of pig origin and, in theory, could have FSH-like activity in some species and not others. Equine chorionic gonadotrophin, which is mostly LH-like in its activity in horses, is considered to have primarily FSH-like activity in rodents and ruminants (Kumar et al., 2010). However, the same pig LH preparation has LH-like activity in cattle (Ribeiro et al., 2007) induced estrus in female dogs by administering 20IU of eCG, once daily for five days, along with 500IU of hCG on the fifth day, and in the group of animals in spontaneous estrus.

With vaginal cytology, they noted that the cell cornification peak occurred earlier in animals subjected to estrus induction, when compared to animals undergoing natural estrus, what did not happened in their work. (Rezende et al., 2018) concluded that intramuscular administration of buserelin acetate was effective in inducing estrus, when administered in two doses on average 10 days apart, allowing fertilization through natural mating. Group 2 animals exhibited proestrus bleeding (5/10) during subsequent breeding season and were subjected to vaginal cytology twice (i.e. first at 4 days after start of bleeding and second next day after the end of bleeding). These animals were advised for mating when $>80 \%$ superficial cells were observed in vaginal cytology.

Following treatment, five and four animals conceived respectively in groups 1 and 2 . It is concluded that both hormonal and nutritional approaches are equally good in inducing fertile estrus in canine.

\section{References}

Arnold, S., Arnold, P., Concannon, P. w., Weilenmann. 1989. Effect of duration of PMSG treatment on induction of oestrus, pregnancy rates and complication of hyper- estrogenism in dogs. Journal of Reproduction Fertility Supplementation. 39:115-122.

Clark, R.D., Stainer, J.R., 1983. Medical and genetic aspects of purebred dogs. In: Edwardsville, Kansas, Veterinary Medicine Publishing Company.

Concannon, P.W., 2002. Methods for induction of estrus in dogs using gonadotropins, $\mathrm{GnRH}$ or dopamine agonists. World Small Animal Veterinary Association congress, Granada, Spain.

Kumar, A.G., Praseeda, R., Rajankutty, k., Alex, P. C., 2010. Comparative efficacy of high vs low dose cabergoline treatment regimen in inducing fertile estrus in anestrus dog. Journal of Animal and veterinary Advances. 9: 1735-38.

Kutzler, M.A., 2005. Induction and synchronization of estrusin dogs. Theriogenology. 64(5): 766-775.

Perkins, N.R., Thomas P.G.A., 1993. Infertility in the bitch with abnormal oestrus cyclicity. Australian Veterinary Practitioners. 23: 122-126.

Peterson, M.E., Melián, C., Nichols, R., 1997. Measurement of serum total thyroxine, triiodothyronine, free thyroxine, and thyrotropin concentrations for 
diagnosis of hypothyroidism in dogs. Journal of American Veternary Medical Association. 211: 1396-1402.

Rezende, R. S., Eurides, D., Barbosa, C. P., Larceda, M. S., Sampoorn, R. L., Gomas, A. L., 2018. Use of GnRH synthetic analog (buserelin) for oestrous induction in female dogs. Arquivo Brasiterio De Medicina Veterinaria e Zootecnia. 70(3): 656-60.

Ribeiro, A.P.C., Vicente, W.R.R., Santos, I.W., 2007. Gonadotrofinas na indução do estro em cadelas. Revista Brasileriade Saúde e Prodcao Animal. 8: 324-334.

Rosychuk, R., 1983. Management of hypothyroidism. In: Current Veterinary therapy. VIII. Editions R.W Kirk. W.B. Sauders, Philadelphia. Pp. 869-875.

Sontas, H. B., Ilse, S., Somi, S. S., 2014. Primary anestrus due to dietary hypothyroidism in a miniature Pinicher bitch. Canadian Veterinary Journal. 55(8):781-785.

Van-Haften, B., Dieleman, S. J., Okkens, A. C., Bevers, M. M., Willemse, A. H., 1989. Induction of oestrus and ovulation in dogs by treatment with PMSG and/Or bromocriptine. Journal of Reproduction Fertility Supplementation. 39: 330-331.

Versetegen, J., Onclin, K., Silva, L. D. M., Concannon, P., 1997. Termination of obligate anestrus and induction of fertile ovarian cycle in dogs by administration of purified pig LH. Journal of reproduction Fertility. 111: 35-40.

Wright, P. J., 1982. The induction of oestrus in the bitch using daily injection of pregnant mare serum gonadotropin. Australian Veterinary Journal. 59:123124.

\section{How to cite this article:}

Honparkhe, M., Jugraj Singh Mahal and Ajeet Kumar. 2020. Estrus Induction by Hormonal and Nutritional Approaches in Canines. Int.J.Curr.Microbiol.App.Sci. 9(05): 1814-1818. doi: https://doi.org/10.20546/ijcmas.2020.905.206 